\title{
(2) OPEN ACCESS \\ Can video consultations replace face-to-face interviews? Palliative medicine and the Covid-19 pandemic: rapid review
}

\author{
Anna Elizabeth Sutherland (D , ${ }^{1}$ Jane Stickland, ${ }^{1}$ Bee Wee ${ }^{2}$
}

- Additional material is published online only. To view please visit the journal online (http://dx.doi.org/10.1136/ bmjspcare-2020-002326)

${ }^{1}$ Department of Palliative Medicine, Sir Michael Sobell House Hospice, Oxford, UK ${ }^{2}$ Department of Palliative Medicine, Oxford University Hospitals NHS Trust, Oxford, Oxfordshire, UK

\section{Correspondence to}

Dr Anna Elizabeth Sutherland, Palliative Medicine, Sir Michael Sobell House Hospice, Oxford OX3 7LE, UK;

annasutherland@doctors.org.uk

Received 26 March 2020 Accepted 16 April 2020 Published Online First 26 May 2020

\begin{abstract}
During the Covid-19 pandemic, a strategy to minimise face-to-face (FtF) visits and limit viral spread is essential. Video consultations offer clinical assessment despite restricted movement of people.

We undertook a rapid literature review to identify the highest currently available level of evidence to inform this major change in clinical practice. We present a narrative synthesis of the one international and one national guideline and two systematic reviews-all published within the last 18 months.

The global evidence appears to support video consultations as an effective, accessible, acceptable and cost-effective method of service delivery. Organisations must ensure software is simple, effective, reliable and safe, with the highest level of security for confidentiality. Although video consultations cannot fully replace FtF, they can radically reduce the need for FtF and the risk of Covid-19 spread in our communities while maintaining high standards of care. For patient safety, it will be critical to follow the WHO guidance regarding: a standard operating procedure; clinical protocols for when video consultations can (and cannot) be used; policies to ensure equity of access in disadvantaged populations; adequate staff training; and administrative support to coordinate appointments.
\end{abstract}

\section{INTRODUCTION}

Due to the rapidly evolving Covid-19 pandemic, a strategy to minimise face-toface $(\mathrm{FtF})$ visits to limit the spread of the virus is essential. Scheduled home visits were reassessed, and telephone proactive support was used where possible. These proactive advice calls were to supplement reactive telephone triage advice line support already available to patients and carers 7 days a week. However, the limitations of telephone assessments meant that frequently FtF home visits were still necessary. As the pandemic progressed, the need for an alternative to further minimise $\mathrm{FtF}$ consultations and capable of overcoming the limitations of telephone advice became urgent.

'Attend Anywhere' has been developed by National Health Service Scotland for this purpose and was identified as an easily accessible, secure video software designed for use as a consultation host for clinicians. The community palliative care team was trained in the software and now use it to assess patients via any internetenabled device owned by the patient or relative and capable of hosting 'Google Chrome' (Alphabet Incorporated) or “'Safari' (developed by Apple).

\section{What is telehealth?}

The WHO states that 'Telehealth involves the use of telecommunications and virtual technology to deliver health care outside of traditional health care facilities'. ${ }^{1}$

The Health Resources and Service Administration also include education in their definition: 'the use of electronic information and telecommunication technologies to support long-distance clinical health care, patient and professional health-related education, public health, and health administration. Technologies include video conferencing, the internet, store-and-forward imaging, streaming media, and terrestrial and wireless communications'. ${ }^{2}$

The term 'telehealth' can encompass many forms of technology, including but not limited to: remote health monitoring; education and training; and telephone and video consultations. However, our rapid review focused specifically on video 
consultations in palliative care, as this is a high priority during the pandemic.

\section{OBJECTIVES}

We undertook a broad literature search to identify all potentially relevant research about video consultations in palliative care. We accepted any comparison (such as usual care or telephone advice), any outcome and any study design including randomised and nonrandomised studies.

\section{METHODS}

Supported by an information specialist, we did a literature search to identify studies of any type, in any language, about telemedicine in palliative care. We searched MEDLINE and Embase on 17 March 2020 (see online supplementary appendix 1 for the full search strategy). Due to the rapid review, a separate protocol was not formally published on a systematic review database such as PROSPERO. Two authors (AES and JS) then screened titles and abstracts independently.

\section{Inclusion criteria}

Studies of any type, other than opinion or editorial articles about video consultations in paediatric or adult palliative care, in any diagnosis were included. We included any comparison, for example: usual care, outpatient appointments and telephone advice.

We did not stipulate primary and secondary outcomes. Instead, we aimed to identify the highest level evidence available both nationally and internationally in order.

We used the Oxford Centre for Evidence Based Medicine guidance on Levels of Evidence ${ }^{3}$ to identify Level 1a Systematic Reviews (in addition to relevant national and international guidelines). We rapidly completed a narrative synthesis of only the highest level documents to facilitate a rapid transformation of this evidence into practice, necessitated by the unprecedented clinical urgency.

\section{RESULTS}

Our search returned 105 records(figure 1). Forty-two records were of relevance at title review. Thirty-four records met or potentially met the inclusion criteriatwo guidelines and two systematic reviews of direct relevance, published within the last 18 months. We summarise their key findings and direct readers to the primary documents for further details.

\section{International guidance on telemedicine}

The WHO recently highlighted the critical part palliative care plays in the international response to humanitarian crises, like the Covid-19 pandemic, to alleviate suffering and care for the dying. ${ }^{4}$

The WHO recommends 'client-to-provider telemedicine: under the condition that it complements, rather than replaces, face-to-face delivery of health services;

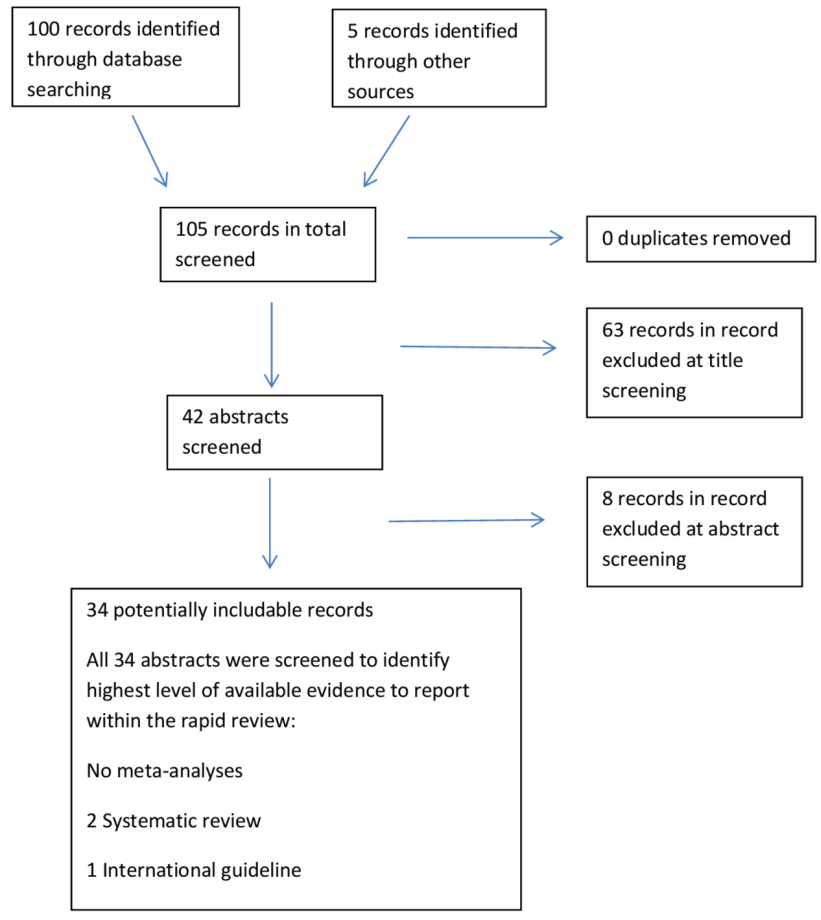

Figure 1 PRISMA diagram. PRISMA, Preferred Reporting Items for Systematic Reviews and Meta-Analyses.

and in settings where patient safety, privacy, traceability, accountability and security can be monitored'. To achieve this, the WHO recommends implementation of 'standard operating procedures and mechanisms to ensure patient safety, privacy, traceability'. The WHO highlights the critical role of the 'accountability of services ... to mitigate the potential risks and harms of implementing this recommendation'.

Additionally, the WHO recommends clear 'clinical protocols to explain what can and cannot be done in the remote consultation'. 5

They also advise particular care be taken to ensure video consultation technology does not further disadvantage underserved and hard-to-reach groups. Special care is necessary to ensure services do not overlook 'people with low literacy or few digital literacy skills, people with limited control over or access to mobile devices, people speaking minority languages, migrant populations in new settings, and people with disabilities such as sight or hearing impairment'. ${ }^{5}$

While remote video consultations may offer a huge benefit to healthcare delivery during the pandemic, the WHO cautioned against rapid implementation without full assessment of the evidence base for the model of care:

'Amid the heightened interest, digital health has also been characterized by implementation rolled out in the absence of a careful examination of the evidence base on benefits and harms. The enthusiasm for digital health has also driven a proliferation of short-lived implementations and an overwhelming diversity of 


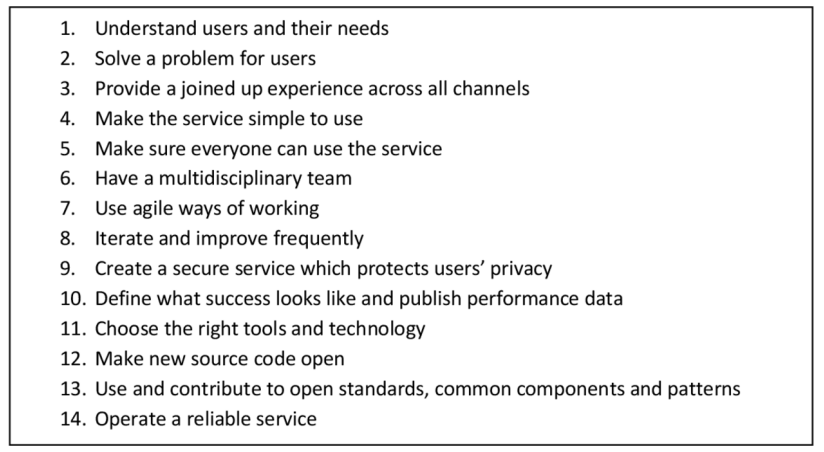

Figure 2 UK Digital Service Standard. ${ }^{6}$.

digital tools, with a limited understanding of their impact on health systems and people's well-being. While recognizing the innovative role that digital technologies can play in strengthening the health system, there is an equally important need to evaluate their contributing effects and ensure that such investments do not inappropriately divert resources from alternative, non-digital approaches'. ${ }^{5}$

We therefore undertook a rapid review of the evidence base about video consultation models in palliative care before implementation.

\section{UK guidelines}

Further to the WHO, the UK Digital Service Standard sets out our duties as health professionals digitally delivered healthcare(figure 2)For further information, please refer to the guidance directly. ${ }^{6}$

\section{What is the level of available evidence for video consultations in delivering palliative care globally?}

We identified one systematic review that addressed this question. A group in Denmark undertook a systematic review in 2019 using an integrative approach. ${ }^{6}$ They defined video consultations as 'real-time interactions between patients and/or relatives and health care professionals that take place via video, thereby allowing participants to have audio/visual contact'? They identified 39 studies world wide of varying designs: mixed methods (14), qualitative (10), quantitative (10), including one randomised control trial and case studies (5). The review included data from 2345 patients, 549 relatives, 252 healthcare professionals and 1631 video consultations. They represented seven different countries worldwide: USA (21); Australia (7); Canada (4); Netherlands (3); UK (2); Brazil (1); and Japan (1). Thirty studies involved specialist palliative care; 5 both specialist and generalist; and $4 \mathrm{FtF}$ generalist palliative care.

Most studies used video consultations to supplement existing palliative care services (27 studies) or compared video consultations to usual care (8 studies), while three reported video consultations as the only palliative care service offered.

Studies used different technology for video consultations: videophones (nine studies), computers with an integrated or external webcam (eight studies), tablets (five studies) and videoconference equipment (three studies). All but one used equipment provided by the healthcare team to the patient and/or relative.

They identified that video consultations broke down the geographical and physical barriers to palliative care access and enabled other healthcare professionals or family members to be present with the patient during consultations. Both relatives and healthcare professionals reported positive experiences and attitudes towards video consultations. The review identified that diagnosis and age were not barriers to video consultations. Importantly, 'several studies described how the visual component of video consultations made it possible for health care professionals in general, specialized, and integrated palliative care, to conduct clinical assessments and control the symptoms and condition of home-based patients and relatives. For example, ... [it] enabled palliative care professionals to notice patients' physical progress or regression such as weight loss or blushing, as well as their mental state, for example, if they had delirium or anxiety'. ${ }^{7}$ They reported that this avoided hospital admissions, influencing place of death. There was uncertainty about whether video consultations could entirely replace $\mathrm{FtF}$ visits. This was particularly true of the initial assessment, especially if there were uncontrolled symptoms or additional paperwork.

Some studies reported professional reluctance with the concept, concerns about technological challenges and potential barriers with frail patients. Additionally, some worried about or avoided sensitive topics because the use of touch was impossible during video consultations. They also identified the need for administrative support in setting up consultations. Without this, some reported video consultation could become time consuming. Additionally, the presence of multiple healthcare professionals during the consultation could be overwhelming for families.

All the 15 different systems had issues with audio, video or internet on occasions. These could be disruptive, although professional training beforehand had a positive effect in mitigating the impact of this. Patient and family familiarity with the device also lessened technological issues.

Despite all but one of the included studies providing patients with the technology to access video consultations, three studies in adults noted a cost reduction compared with outpatient appointments or inpatient admission. Patients also reported less outlay in travel, cost and time, with more patients accessing palliative care services than outpatient based hospitals appointments.

What is the level of available evidence for remote consultation software in palliative care in the UK?

We identified two systematic reviews that addressed this. ${ }^{89}$ A paper in 2019 reported an update an original 
2010 review. Telemedicine has the potential to improve access to palliative care particularly in remote and rural areas, reducing the inequity in specialist palliative care availability currently observed in the UK. ${ }^{9}$ They stated: 'Telehealth may provide a solution to meeting the growing demands of palliative care services across geographical regions with limited resources. Ready access to general and specialist palliative care services for patients with a variety of life-limiting conditions may prove beneficial in reducing the need for emergency services'.

The 2019 systematic review identified 30 studies of telehealth interventions in palliative care in the UK. However, none included video consultations for direct patient care. Models identified included electronic patient records, telephone advice consultations and telehealth monitoring (eg, oxygen saturations, respiratory rate, blood pressure, heart rate and weight). ${ }^{9}$

\section{DISCUSSION}

Recent Italian experience suggests we cannot entirely avoid $\mathrm{FtF}$ visits during the pandemic but minimise this to reduce exposure for ourselves and our vulnerable patients. When home $\mathrm{FtF}$ visits are unavoidable, we should:

1. 'Discuss and share with colleagues the cases of families at home to make better decisions about care and share the weight of these decisions.

2. Visit the home with appropriate PPE, ${ }^{10}$

Despite the positive response from patients and relatives when video consultations are done as an alternative to $\mathrm{FtF}$, there is reluctance by some professionals to accept them as an adequate mode of service delivery. In normal clinical practice, this might lead to a paced introduction and testing of new work methods; however, this is unlikely to be feasible during a pandemic. Supporting staff during this transition will be critical to reduce the stress inherent in such a rapid practice change. The article 'Top Ten Tips Palliative Care Clinicians Should Know about Telepalliative Care $^{11}$ offers practical suggestions for professionals undertaking a transition to video consultation, and may help support staff.

While various technical difficulties will inevitably occur, we will have to bear with this to deliver high level specialist and generalist palliative care. Staff training is critical to minimise the anxiety and frustration this may bring, as well as encouraging patients and relatives to access video consultation using familiar hardware.

Nevertheless, without video consultations, community palliative care services will be restricted to telephone advice with inherent limitations. In particular, the risk of limited assessments and increased hospital admission rates would put vulnerable populations at risk of Covid-19 during their admission and further increase healthcare service demands when it can least accommodate it. Every palliative care service should consider how best to meet the needs of their community and maximise the benefits of modern technology while minimising the risk of viral transmission.

To facilitate this rapid review, we summarised key findings of the identified systematic reviews without further critical appraisal, enabling readers to undertake further analysis of these primary sources. We elected to only search Medline and Embase meaning that other relevant studies, in particular unpublished studies and grey literature, may have been missed.

While completion a full systematic review might have led to the identification of new articles and new evidence, there was insufficient time to do this due to the urgency of the clinical question.

Furthermore, by limiting this review to only palliative care, we may have omitted transferrable learning from other disciplines.

\section{CONCLUSIONS}

Although video consultations cannot fully replace FtF encounters, it may radically reduce the need, so combating the risk of spreading Covid-19 further in our communities. While video consultations for palliative care in the UK will be a radical change in service delivery, the international evidence appears to support it as effective, accessible, acceptable and cost-effective. Importantly, patients and relatives report video consultations to be highly acceptable and often wish it had been offered sooner. The principle concern from health professionals is most notably in relation to the confidentiality and security of the video consultations.

It is imperative (in line with the UK Digital Services Standard), that organisations ensure software is simple, effective, reliable and safe, with highest level of security always embedded to ensure the confidentiality of patients. For patient safety during the introduction, it will be critical for every service to follow WHO guidance by having: a standard operating procedure; clinical protocols stipulating when video consultations can and cannot be used; policies to minimise the risk of reduced equity of access in disadvantaged populations; adequate staff training; and administration support to coordinate appointments. These safeguards will allow us to focus on clinical care and minimise any potential disruption in clinical practice.

Contributors JS suggested the article and request the literature search. AES and JS independently screened titles and abstracts. AES wrote the final manuscript with supervision, comments and review from JS and BW.

Funding The authors have not declared a specific grant for this research from any funding agency in the public, commercial or not-for-profit sectors.

Competing interests None declared.

Patient consent for publication Not required.

Provenance and peer review Not commissioned; internally peer reviewed.

Open access This is an open access article distributed in accordance with the Creative Commons Attribution Non 
Commercial (CC BY-NC 4.0) license, which permits others to distribute, remix, adapt, build upon this work noncommercially, and license their derivative works on different terms, provided the original work is properly cited, appropriate credit is given, any changes made indicated, and the use is noncommercial. See: http://creativecommons.org/licenses/by-nc/4. $0 /$.

\section{ORCID iD}

Anna Elizabeth Sutherland http://orcid.org/0000-0001-77812099

\section{REFERENCES}

1 WHO. Health and sustainable development, 2020. Available: https://www.who.int/sustainable-development/health-sector/ strategies/telehealth/en/ [Accessed 23 Mar 2020].

2 HRSA. Telehealth programs. Available: https://www.hrsa.gov/ rural-health/telehealth [Accessed 23 Mar 2020].

3 CEBM. Levels of evidence, 2009. Available: https://www.cebm. net/2009/06/oxford-centre-evidence-based-medicine-levelsevidence-march-2009/ [Accessed 26 Mar 2020].

4 WHO, 2018. Available: https://reliefweb.int/sites/reliefweb. int/files/resources/9789241514460-eng.pdf [Accessed 21 Mar 2020].
5 WHO. WHO guideline recommendationson digital interventionsfor health system strengthening, 2019. Available: https://apps.who.int/iris/bitstream/handle/10665/ 311941/9789241550505-eng.pdf?ua=1 [Accessed 21 Mar 2020].

6 GOV. Coronavirus (COVID-19): what you need to do, 2019. Available: https://www.gov.uk/service-manual/service-standard [Accessed 21 Mar 2020].

7 Jess M, Timm H, Dieperink KB. Video consultations in palliative care: a systematic integrative review. Palliat Med 2019;33:942-58.

8 Kidd L, Cayless S, Johnston B, et al. Telehealth in palliative care in the UK: a review of the evidence. J Telemed Telecare 2010;16:394-402.

9 Hancock S, Preston N, Jones H, et al. Telehealth in palliative care is being described but not evaluated: a systematic review. BMC Palliat Care 2019;18:114.

10 Valenti D. From the front line: palliative care in bologna during the COVID-19 crisis, 2020. Available: https://eapcnet. wordpress.com/ [Accessed 2403 2020].

11 Calton BA, Rabow MW, Branagan L, et al. Top ten tips palliative care clinicians should know about Telepalliative care. J Palliat Med 2019;22:981-5. 